Prof. Dr. Peter Berlit

Deutsche Gesellschaft für Neurologie (DGN)

Reinhardtstr. 27C, 10117 Berlin, Deutschland

berlit@dgn.org

\title{
Interessenkonflikte - ein wichtiges Thema für die Deutsche Gesellschaft für Neurologie (DGN)
}

Bekanntermaßen beschäftigt sich die Deutsche Gesellschaft für Neurologie (DGN) seit vielen Jahren mit Interessenkonflikten („conflicts of interest“ [COI]). Bei den Leitlinien (LL) nimmt unsere Fachgesellschaft dabei eine Vorreiterrolle ein. Eigene SOP („standard operating procedures“) zur Bewertung von Interessen wurden von uns definiert, getestet und in den Regelbetrieb überführt.

Bevor eine Arbeitsgruppe ihre Tätigkeit aufnimmt, erfolgt die Fremdbewertung der Interessen durch anonym arbeitende, unabhängige und sachkundige Interessenkonfliktbeauftragte (IKB) der DGN, die aufgrund der jeweils dargelegten Interessen ihre Empfehlungen aussprechen. Die Konsequenzen sollten unter Abwägung des potenziellen Nutzens (z. B. Erhalt der Glaubwürdigkeit) und des möglichen Schadens (z. B. Verlust von Expertise) intensiv und kritisch innerhalb der LL-Gruppe diskutiert werden. Dabei kommen Fragen nach finanziellen Schwellenwerten auf und danach, ob Gelder an die Klinik/Institution des LL-Autors oder an ihn persönlich geflossen sind. Auf der einen Seite erscheinen Interessenkonflikte anhand von finanziellen Schwellenwerten leichter standardisierbar, vergleichbar etwa zur Transparenzregelung für Bundestagsabgeordnete. Andererseits muss aber bedacht werden, dass letztlich das Risiko unabhängig von der Höhe des Betrags ist, die Angemessenheit einer Gegenleistung nicht differenziert wird und indirekte Interessen unbedingt stärker berücksichtigt werden sollten. Bei den meisten Konstellationen hilft bei der Wertung und Einschätzung ein einfacher Grundsatz weiter:

Ein COI ist eine Risikokonstellation, nicht ein nachweisbares Fehlverhalten, und wenn mein Geldgeber ein Interesse an der Richtung meiner Empfehlung hat, besteht für mich ein Interessenskonflikt.

Aus der Bewertung der COI durch die IKB und die LL-Gruppe resultieren für LL-Mitglieder 3 Handlungsmöglichkeiten:

1. keine Konsequenz,

2. Stimmenthaltung bei Abstimmungen oder

3. Ausschluss aus der Gruppe

Das bedeutet: Ein Autor mit hohem COI kann bei ausgewogenem Redaktionskomitee an der LL mitwirken. Allerdings sind in

DGNeurologie 2019 • 2 (3): 149

https://doi.org/10.1007/s42451-019-0076-0

๑) Springer Medizin Verlag GmbH, ein Teil von Springer Nature 2019 diesem Fall nur schriftliche Stellungnahmen möglich, jedoch keine Teilnahme an Beratungen der LL-Gruppe und keine Mitwirkung an Abstimmungen (dies entspricht der Regel der AWMF [Arbeitsgemeinschaft der Wissenschaftlichen Medizinischen Fachgesellschaften e. V.] zum Umgang mit Interessenkonflikten, https://www.awmf.org). Die Umsetzung beschlossener Maßnahmen, wie Enthaltungen oder Mitwirkung nur an bestimmten Kapiteln, obliegt dem Koordinator. Nach geltenden Regeln von AWMF und DGN soll dieser keine oder nur geringe Interessenkonflikte ohne thematischen Bezug haben. Anderenfalls zieht die DGN einen Kokoordinator ohne COI hinzu. Transparenz über die Ergebnisse der Diskussion und deren Dokumentation ist hier von maßgeblicher Bedeutung. Erforderlichenfalls wird die Arbeitsgruppe neu zusammengesetzt. Die 50 \%-Regel der DGN muss in jedem Fall eingehalten werden: Die Hälfte der Arbeitsgruppe darf keine oder nur geringe, für die LL nicht relevante Interessenkonflikte haben. Wie ein Aufruf in der DGN zeigte, gibt es speziell auf Oberarztebene eine Vielzahl von kompetenten Neurologen, die keine COI haben und gerne an LL mitarbeiten.

Die Ergebnisse der umgesetzten Maßnahmen können Sie als Leser und Nutzer unserer Leitlinien auf der Homepage der DGN jeweils im LL-Abschnitt „Erklärung von Interessen und Umgang mit Interessenkonflikten“ einsehen. Das gilt auch für die LL, die Sie in diesem Heft der DGNeurologie finden.

Und was für Leitlinien gilt, sollte auch für die Weiterbildungsinhalte von Zeitschriften gelten. In diesem Heft der DGNeurologie finden Sie die Erklärung zu finanziellen und nichtfinanziellen Interessen bei CME-Fortbildungen (CME: „continuing medical education"), die jetzt zwingend vorsehen, dass auch unsere CMEHerausgeber ihre Interessenskonflikte offenlegen. Dies ist sicher ein Schritt in die richtige Richtung.

Denn dafür stehen wir als DGN mit unseren Leitlinien und mit unserer Zeitschrift: für aktuelle, kompetente, v. a. aber unabhängige und neutrale Fachinformationen und Handlungsanweisungen.

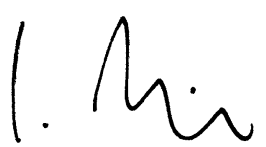

Ihr Peter Berlit

Interessenkonflikt. P. Berlit gibt an, dass kein Interessenkonflikt besteht. 